\title{
Investigation of Quality of Experience for 3D Video in Wireless Network Environment*
}

\author{
Ivett Kulik and Tuan Anh Trinh \\ Budapest University of Technology and Economics, \\ Department of Telecommunications and Media Informatics, \\ Budapest, Hungary \\ \{kulik, trinh\}@tmit.bme.hu
}

\begin{abstract}
Multimedia streams have increased dramatically over the wireless networks during the last few years. 3D video stream-based applications and movies will become significant contents of wireless network traffic in future Internet. This paper shows Quality of Experience (QoE) results based on transport network's Quality of Service (QoS) level degradation obtained from subjective tests which were carried out for 3D stereoscopic video files transporting through GPON-based transport network combined with IEEE802.11n standard based WiFi sub-networks on clients' side. Gathered information show that QoE level is influenced with more factors like: bandwidth limitation or channel interference in WiFi network and QoS parameters' values of transport network as well. In case of 3D contents quality of film shooting, size and resolution value of using display also take effect on perceived visual quality. Evaluation results were processed statistically IBM SPSS Statistics software-package.
\end{abstract}

Keywords: Quality of Experience (QoE), Quality of Service (QoS), Gigabit Passive Optical Network (GPON), WiFi network, 3D stereoscopic video, subjective test, IBM SPSS.

\section{Introduction}

Widespread deploying of wireless local area networks (WLAN) brings on facilitation for customers. They can connect more easily to the Internet and the number of Internet users has increased rapidly. WLAN based on IEEE 802.11 standards with infrastructure mode is the most popular as we can recognize hot spots everywhere. Popularity of multimedia contents - especially 3D based video-games or movies - has raised the necessity of this technology progress. Today the IEEE 802.11n standard based communication support advanced multimedia applications but nature of wireless technology (like bandwidth limitation or channel interference) still brings to challenging issues for Internet Service Providers (ISPs). Customers' satisfaction needs more than only QoS support on the part of service providers, enhanced level of vision quality is also very important which is called Quality of Experience (QoE).

* The research was supported by NKTH-OTKA grant CNK77802. 
More research subjects had brought into focus the QoE and QoS [4], [10] or evaluation of 3D stereoscopic images and films [6], [7], [8] but nobody had published specific results of subjective tests carried out by bigger number of participants $(30-50$ testing persons) focusing to relationship between QoE and QoS in case of 3D stereoscopic videos. We made first evaluation of QoE for 3D stereoscopic video files based on QoS through the GPON-based transport network with 50 participants, which results were published in paper [1]. Our research results showed that in the investigated scenarios GPON is suitable for efficient transport of 3D contents. QoE results of 3D stereoscopic videos' watching showed dependency on the transport network caused QoS degradations but more research was needed for observing and improving quality of user experience in case of multimedia contents delivery. Therefore we carried out further subjective tests with 40 participants through GPON-based transport network but with more features investigation and more specific results were obtained. Finally we collected information from people (36 participants) in GPON environment combined with WiFi network on the clients' side with reason to explore QoE-QoS relationship is this case. The latter scenario is discussed in this paper. Evaluation results have been analyzed by IBM Statistical Package for Social Scientists (SPSS).

The paper is structured as follows. Section 2 shortly describes methods of quality comparisons and some statistics analysis definitions. Section 3 shortly shows used topology of laboratory network. Section 4 presents description of experiment and evaluated results of measurements through the GPON transport network combined with WiFi network. Finally we conclude the paper in Section 5.

\section{Methods of Quality Comparisons}

When a stereoscopic film is viewed the resulting experienced is formed by many factors. Some factors related to stereoscopic materials, cognitive-emotional factors related to the viewer, the viewer's visual limitations and the viewing content. The technology that is used to watch the materials and the display's size and resolution has also significant impact on the viewer's experience. Values of transport network's QoS parameters like delay, jitter, packet loss and throughput could have also important effect to the visual quality results and multimedia attributes like coding, bit-rate, framerate and motion level of scenes influence results as well [10]. This means that the investigation of QoE besides the importance is a complicated process and the correct evaluation of gathered information is also a challenge.

The derivation of QoE-QoS relationships builds on quality comparisons between:

- The reference - which is the undistorted video stream

- The outcome - which is the potentially distorted video stream due to the QoS degradation.

References play an important role when it comes to rating the quality of outcome. There are two basic measurement options: subjective tests and objective tests [10].

Subjective tests are carried out by real users and this type of test is time consuming because a large number of people have to participate on it for statistically relevant results. Objective tests are carried out by an algorithm following psychophysical and engineering approaches without human presence. 
QoE-QoS relationship can be investigated based on full reference metrics - which allow detailed subjective and objective comparisons of multimedia contents because both reference and outcome are available or based on no reference metrics - which is an online situation where no reference is available and quality results can be extracted only from the outcomes finally based on reduced reference metrics - which is not so detailed comparison than the case of full reference metrics but here the same set of parameters are derived and compared for the reference and outcome as well [10].

Multimedia sequences (undistorted and distorted contents as well) can be scored by the Mean Opinion Score (MOS) in case of subjective evaluation, which is the core of our experiment. The MOS quality scale method used to be applied for voice and video traffic scale. Scores are: 5 - excellent, 4 - good, 3 - regular, 2 - bad, 1 - awful.

\subsection{Statistics Analysis}

Collected data were analyzed by the IBM SPSS Statistics software-package for business, government, research and academic organizations. SPSS is an abbreviation for Statistical Package for Social Scientists but the other name: Statistical Product and Service Solutions are also well-known. This software offers statistical procedures to make reliable analysis, to get a quick look at our data, formulate hypotheses for additional testing, and then carry out a number of procedures to help clarify relationships between variables, create clusters, identify trends and make predictions. The goal of analysis is to make correct judgments and decisions in the presence of uncertainty and variation and to detect relations between data [14]. Statistics methodology is founded on the probability study. A study is an experiment if the investigator observes how a response variable behaves when one or more explanatory variables, also called factors, are manipulated. In our experiments QoE was the response variable scored by the MOS and QoS parameters like jitter degradation and bandwidth limitation were explanatory variables.

The method of least squares where also used, which is a standard approach to the approximate solution for sets of equations in which there are more equations than unknowns. "Least squares" means that the overall solution minimizes the sum of the squares of the errors made in solving every equation.

A confidence interval is a particular kind of interval estimate of a population parameter and is used to indicate the reliability of an estimate. It is an observed interval that frequently includes the parameter of interest, if the experiment is repeated. How frequently the observed interval contains the parameter is determined by the confidence level or confidence coefficient [14].

\section{Topology of Laboratory Network}

Experiments were carried out in laboratory environment where the same GPON-based transport network was used as core of the network topology which is described in the paper [1]. This topology was increased with two WiFi sub-networks where WiFi Access Points (AP) were connected directly to ONTs (Optical Network Terminal) and two clients' PCs received 3D stereoscopic video files through WiFi network cards. The topology is shown in Figure 1. 


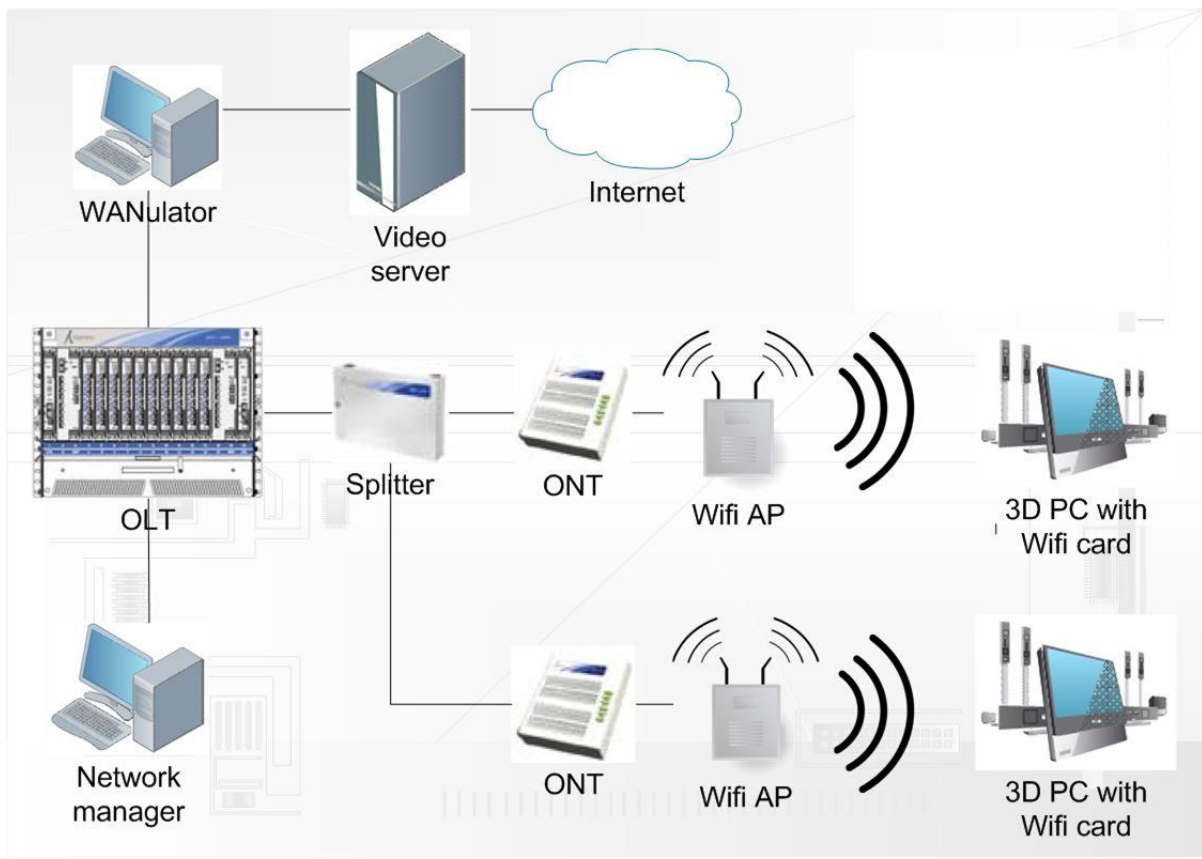

Fig. 1. The GPON-based + WiFi sub-networks topology

Hardware configurations of transport network's devices, multimedia server and clients are also mentioned in [1]. Types of used Access Points were: Linksys E4200 Dual-Band N Router and Linksys WRT160NL-EE Router which were connected to $100 \mathrm{Mb} / \mathrm{s}$ Ethernet port of ONTs. PCs' displays were Samsung Syncmaster 2233 with screen size 22"and resolution 1920 x 1080 and we used WiFi cards: TP Link TL-WN95IN.

Video contents had to be transfer through the transport network only in unicast mode instead of multicast mode because the VLC media player v2.0 could not play 3D stereoscopic content and Nvidia Vision Player v1.6 (NVP) could play correctly 3D stereoscopic video files but only streams transported only in unicast mode and based on TCP transport protocol.

\section{Description of Experiment}

The common practice to estimate user perception is to conduct large experiments in a controlled environment. Based on first and second experiences we prepared new investigation with goal to show relationship between QoE and QoS in laboratory environment extended with WiFi sub-network.

Participants had to evaluate some important points based on MOS like: 1) video continuity, 2) quality of picture, 3) 3D visual quality, 4) conformity between picture and voice and 5) QoE on the whole. The last point is more complicated than only recapitulation of first ones. We also asked people to weight their answer for statistics analysis because we could calculate the weighted average values of QoE based on gathered weighted scores. 


\subsection{Evaluated Results}

36 participants attended this experiment (34 men and 2 women) who study at the Budapest University of Technology and Economics. 18 were spectacled from them and their mean age was 22.14. 32 participants have watched 3D movies. They watched a short part of 3D version of Avatar trailer which general features are shown in Table 1.

Table 1. Features of the investigated 3D stereoscopic video

\begin{tabular}{|c|c|c|c|}
\hline Title & Video codec & Audio codec & Container format \\
\hline Avatar & $\begin{array}{c}\text { WMPv9 (VC-1 } \\
\text { Simple/Main) }\end{array}$ & WMAv2 & wmv \\
\hline $\begin{array}{c}\text { Length } \\
\text { (mm:ss) }\end{array}$ & Resolution & $\begin{array}{c}\text { Avrg. Video } \\
\text { bitrate (kb/s) }\end{array}$ & $\begin{array}{c}\text { Avrg. Audio bitrate } \\
\text { (kb/s) }\end{array}$ \\
\hline $03: 32$ & $1280 * 720$ & 9646 & 192 \\
\hline
\end{tabular}

At first participants watched 3D stereoscopic video without any added disturbance. Despite of using IEEE802.11n standard in the $2.4 \mathrm{GHz}$ band video playing on $2 \mathrm{PCs}$ in same time was not always fluent with standard quality which was caused by two major circumstances. At first many $\mathrm{WiFi}$ networks were accessible in the department laboratory where our experiment was carried out and other WiFi networks used same channels for carrying which is shown in Figure 2 and Table 2.

Table 2. WiFi networks characteristics during the experiment

\begin{tabular}{|c|c|c|c|c|c|}
\hline 16:00 PM & \multicolumn{3}{|c|}{ Channel Usage [\%] } & \multirow{2}{*}{$\begin{array}{l}\text { AP numb. on } \\
\text { channel }\end{array}$} & \multirow{2}{*}{ All APs numb. } \\
\hline Channel & Before & During & After & & \\
\hline 3 & $13-16$ & $49-61$ & $12-15$ & 3 & \multirow{2}{*}{18} \\
\hline 13 & $4-7$ & $48-57$ & $5-7$ & 1 & \\
\hline
\end{tabular}

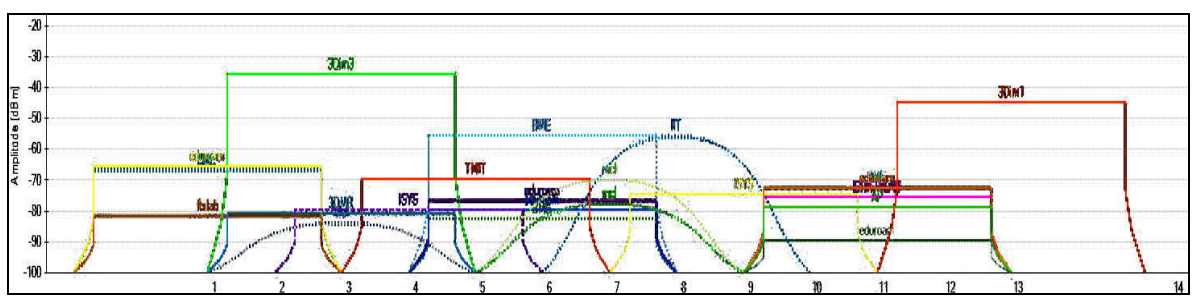

Fig. 2. Active WiFi networks in the laboratory environment

Second important reason was one technical parameter of ONT: 100Mb/s Ethernet port which was directly connected to the AP. This throughput also prevented to get convenient vision quality during the watching. Therefore network topology of 
reference test was reduced to only one ONT, one AP and one PC on the client's side. We carried out reference test measuring with Linksys E4200 Dual Band N Router used channel 3. for IEEE 802.11n standard based connection and other reference test with Linksys WRT160NL-EE router used channel 13. for IEEE 802.11n standard based connection. In first case mean value of result scores was 4.8 (close to excellent) and in second case mean value was 4.5 (between excellent and good. Scores were always recorded by the test persons for 5 important viewpoints mentioned above. NVPv1.6 used and default buffering time during the whole experiment $440 \mathrm{~ms}$.

Other subjective tests with distorted 3D video contents were carried out on these two PCs in same time and each of them was connected to different AP in the way mentioned above. We can say even based on reference tests that $3 \mathrm{D}$ content delivery though the WiFi network is more sensitive and less effective comparing results with first or second investigation results where we could carry out measurements on 4 PCs with mean value of QoE 4.355 (better than good) calculated based on weighted scores. Participants perceived quality of 3D presentation also by quality of stereo shooting like depth of scenes.

For this reason we did not disturb 3D video transport by jitter increase or jitter increase combined with bandwidth limitation but only with bandwidth limitation. We counted weighted averages by formula (1) based on gathered scores and weights.

Formula of weighted average:

$$
\text { Weighted average }=\sum_{i=1}^{5} \frac{\text { Weight }_{i} \text { Value }_{i}}{\text { Sum_of_Weights }}
$$

Where: $i$ - sequential number of the appropriate question point Sum_of_Weights - sum of weights for 3D video.

Based on weighted average values we could assign one QoE value to every appropriate value of bandwidth limitation. Summary of results are shown in Table 3.

Table 3. Summary of Calculated Weighted Values

\begin{tabular}{|c|c|c|c|c|c|c|c|}
\hline \multicolumn{7}{|c|}{ Bandwidth [MB/s] } \\
\hline 3D QoS & $\mathbf{5 . 0}$ & $\mathbf{4 . 7 5}$ & $\mathbf{4 . 5 0}$ & $\mathbf{4 . 2 5}$ & $\mathbf{4 . 0}$ & $\mathbf{3 . 7 5}$ & $\mathbf{3 . 5 0}$ \\
\hline 3D QoE & 3.2167 & 3.1266 & 3.0750 & 3.0521 & 2.9861 & 2.5556 & 2.1250 \\
\hline + conf. interval & 3.5101 & 3.4171 & 3.3518 & 3.3066 & 3.2186 & 2.8042 & 2.3868 \\
\hline - conf. interval & 2.9233 & 2.8608 & 2.7982 & 2.7978 & 2.7536 & 2.3070 & 1.8632 \\
\hline
\end{tabular}


Bandwidth limitation values were calculated based on the average demand bandwidth value of $3 \mathrm{D}$ content which was during the $95 \%$ of playing time around $4 \mathrm{MB} / \mathrm{s}$ except in case of the highest motion level scenes when spine values appeared exceeded this $4 \mathrm{MB} / \mathrm{s}$ bandwidth value. Therefore bandwidth was set to 5, 4.5, 4.0, 3.75 and $3.5 \mathrm{MB} / \mathrm{s}$ which means $0,0.5,1,1.25,1,5 \mathrm{MB} / \mathrm{s}$ limitation values on Figure 3.

Figure 3 shows relationship between QoE degradation and bandwidth limitation's difference increasing by using interpolation lines for 2PCs together (using 3.channel and 13.channel) and for each PC separately.

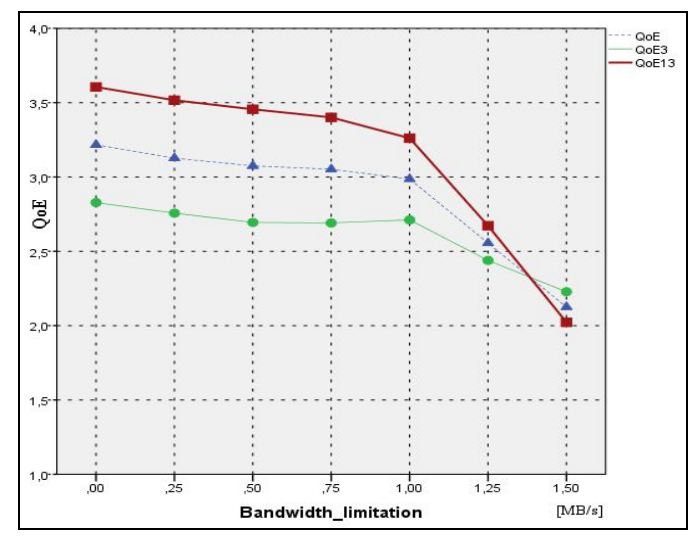

Fig. 3. QoE degradation based on bandwidth limitation

Appropriate mathematical descriptions were calculated by the Wolfram Alpha application. Applying the method of least squares we got solutions:

$$
\begin{aligned}
& \text { 3.channel }+13 . \text { channel: }-0.767565 x^{3}+1.02543 x^{2}-0.550797 x+3.21225 \\
& \text { 3.channel: }-56666 x^{3}+0.94104 x^{2}-0.547423 x+2.82378 \\
& \text { 13.channel: }-0.968302 x^{3}+1.10933 x^{2}-0.553944 x+3.60071
\end{aligned}
$$

This means that QoE-QoS relationship for 3D stereoscopic video watching shows cubic correlation for channel 3 and channel 13. as well. The threshold value of QoS parameter was in case bandwidth $4 \mathrm{MB} / \mathrm{s}$ which means $1 \mathrm{MB} / \mathrm{s}$ bandwidth limitation value on Figure 3.

We can recognize from Figure 3 that 3D film delivering through 13.channel gives better quality results than communication through 3.channel which was used with more different WiFi networks in same time - shown in Table 2. The middle line shows combined results of these two channels information when started mean weighted QoE was only 3.2167 (regular). This was caused by the highest motion level scenes when the data sequences stuck shown in Figure 4 but these critical situations soon were persisted. 


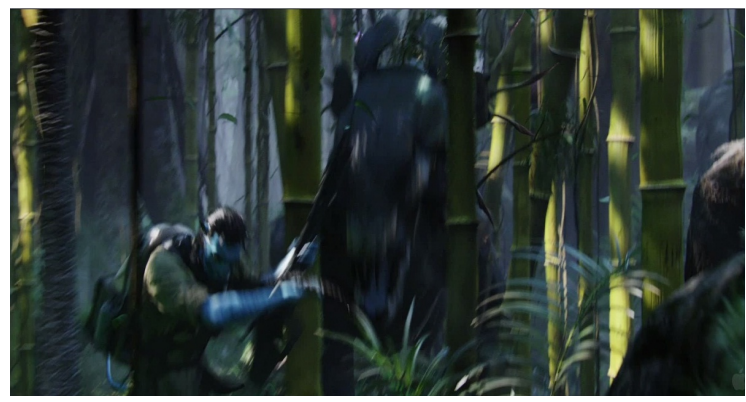

Fig. 4. Data sequences stuck in the highest motion level

In case of confidence intervals (CI) we presupposed normal distribution and $90 \%$ confidence interval and we calculated based on requirements:

Deviation of appropriate variable and the critical value multiplied together gives the CI. Figure 5 in middle shows line of weighted averages of all results (3. channel + 13.channel results) and lines above and under show margins of CI.

QoE sensibility to bandwidth limitation was conspicuous and from the different value from $1 \mathrm{MB} / \mathrm{s}$ rapid deterioration turned up and participants became dissatisfied. This means that bigger limitation than $5 \%$ of demand bandwidth value is not recommended for 3D stereoscopic contents if we want approach excellent or good MOS score of QoE.

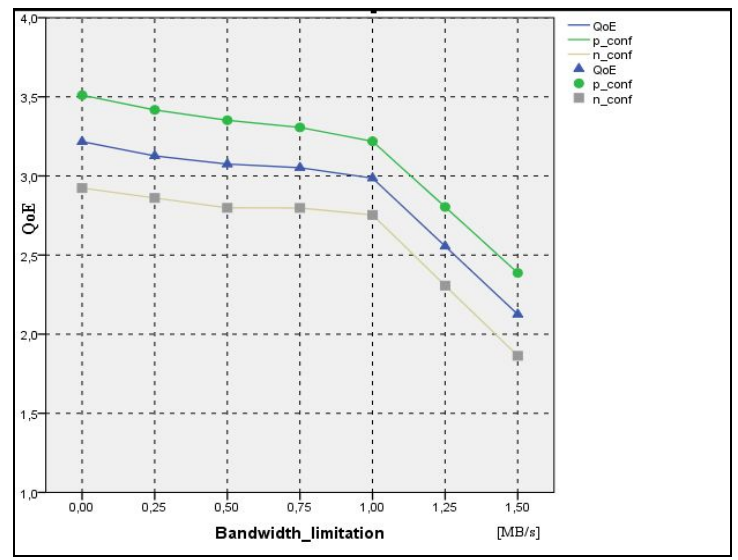

Fig. 5. Confidence intervals of QoE values

Based on Figure 3 and Figure 5 we can conclude that less QoS deterioration cases worse QoE in case of WiFi network using and to supply sufficient throughput is an important characteristic point to satisfy customers' demand. The buffered playing time increase also could help to raise resistivity against QoS degradation [15] but we did not change this value. 


\section{Conclusion}

In this paper we presented results of subjective test method of QoE investigation of 3D stereoscopic video files based on transport network QoS level degradations. In combined network environment GPON-based transport network + WiFi network based on IEEE $802.11 \mathrm{n}$ in the $2.4 \mathrm{GHz}$ band at the client's side the QoE is more sensitive than in case of network topology without WiFi sub-network. Video contents were transported in unicast mode based on TCP protocol and were played on two PCs in the same time. The relationship between QoE and QoS was shown based on gathered data for 3D stereoscopic multimedia content but the good quality guarantee is more complex in case of WiFi network. QoE is influenced by nature of wireless technology (like bandwidth limitation or channel interferences) and by QoS level in transport network as well.

Gathered data show that people who had watched 3D movie before this experiment (32 from 36 participants) were more critical to any quality differences. Evaluation of data was carried out by IBM SPSS Statistics software. Statistic outputs clearly show close coherence between QoE and QoS changing and robustness of 3D contents and limitation of WiFi networks together cause stronger QoE degradation on the client's side. We can emphasize that for good QoE of 3D stereoscopic contents delivery through WiFi network the demanded bandwidth should be provided.

Our results also show that quality of 3D presentation is also strongly influenced by quality of stereo shooting and 3D production. More cameras using (today for 3D movie filmmakers use 10 - 20 cameras with high quality) also causes increase of 3D videostreams robustness. The stereoscopic type of displaying also could case ,visual discomfort" as well. Researchers working in vision and graphics are attempting to develop new techniques and technologies to overcome the current limitations in $3 \mathrm{D}$ stereoscopic presentation.

Primary importance of QoE investigation in wireless transport network environment is came to the front due to the worldwide growing of video-streams presentation on smart but small mobile devices.

Therefore future work will address investigation of gathered information from experiments carried out with smart mobile devices on client sides and all research works results in the subject: investigation of QoE - QoS relationship for 3D stereoscopic videos delivery can be used in future research work. The goal is mathematical modeling of functional relationship between QoE and QoS metrics which is needed for optimal solution of 3D stereoscopic video contents delivery with appropriate display quality.

\section{References}

1. Kulik, I., Trinh, T.A.: Investigation of Quality of Experience for 3D Streams in Gigabit Passive Optical Network. In: Lehnert, R. (ed.) EUNICE 2011. LNCS, vol. 6955, pp. 157168. Springer, Heidelberg (2011)

2. Zahariadis, T., Daras, P., Laso-Ballesteros, I.: Towards Future 3D Media Internet. In: Network \& Electronic Media - Summit, St. Malo France (October 2008)

3. Cale, I., Salihovic, A., Ivekovic, M.: Gigabit Passive Network - GPON. In: ITI 2007 International Conference on Information Technology Interfaces, Cavtat, Croatia, pp. 679684 (June 2007) 
4. Casas, P., Belzarena, P., Vaton, S.: End-2-End Evaluation of IP Multimedia Services, a User Perceived Quality of Service Approach. In: 18th ITC Specialist Seminar of Quality of Experience, Karlskrona, Sweden, pp. 13-23 (May 2008)

5. You, J., Xing, L., Perkis, A.: Quality of Visual Experience for 3D Presentation Stereoscopic. In: Mrak, M., Grgic, M., Kunt, M. (eds.) High-Quality of Visual Experience. Signals and Communication Technology, ch. 3, vol. I, pp. 51-77 (2010)

6. Häkkinen, J., Kawai, T., Takatalo, J., Leisiti, T., Radun, J., Hirsaho, A., Nyman, G.: Measuring Stereoscopic Image Quality Experience with Interpretation Based Quality Methodology. In: IS\&T/SPIE's International Symposium on Electronic Imaging, San Jose, California USA (January 2008)

7. Shibata, T., Kurihara, S., Kawai, T., Takahashi, T., Shimizu, T., Kawada, R., Ito, A., Häkkinen, J., Takatalo, J., Nyman, G.: Evaluation of stereoscopic image quality for mobile devices using Interpretation Based Quality methodology. In: Proc. SPIE, vol. 7237 (2009)

8. Xing, L., You, J., Ebrahimi, T., Perkis, A.: Estimating Quality of Experience on Stereoscopic Images. In: ISPACS 2010 - International Symposium on Intelligent Signal Processing and Communication Systems, Chengdu (December 2010)

9. Lambooij, M., Ijsselsteijn, W., Heynderickx, I.: Visual Discomfort in Stereoscopic Displays: A Review. Journal of Imaging Science and Technology 53(3), 030201-(14) (2009)

10. Fiedler, M., Hossfeld, T., Tran-Gia, P.: A Generic Quantitative Relationship between Quality of Experience and Quality of Service. IEEE Network 24(2), 36-41 (2010)

11. Kirk, K.L.: Looking Beyond Stereoscopic 3D's Revival. Communications of the ACM 53(8), 14-16 (2010)

12. Dai, Q.: A Survey of Quality of Experience. In: Lehnert, R. (ed.) EUNICE 2011. LNCS, vol. 6955, pp. 146-156. Springer, Heidelberg (2011)

13. Fort, S.: 2020 3D Media: New directions in Immersive Entertainment. In: SIGGRAPH 2010 - International Conference and Exhibition on Computer Graphics and Interactive Techniques, Los Angeles, USA ( July 2010)

14. Milton, J.S., Arnold, J.C.: Probability and Statistics in the Engineering and Computing Sciences. McGraw Hill International Editions (1986)

15. Rugel, S., Knoll, T.M., Eckert, M., Bauschert, T.: A Network-based method for measuring of internet video streaming quality. In: 1st European Teletraffic Seminar, Poznan, Polland (February 2011)

16. International Telecommunication Union, "Subjective video quality assessment methods for multimedia applications", rec. ITU-T P.910 (1999)

17. Garroppo, R., Giordano, S., Oppedisano, F., Procissi, G.: A Receiver Side Approach For Real Time Monitoring of IP Performance Metrics. In: Proc. of the EuroFGI Workshop on IP QoS and Traffic Control, pp. 169-176 (2007)

18. ur Rehman Laghari, K., Crespi, N., Molina, B., Palau, C.E.: QoE Aware Service Delivery in Distributed Environment. In: 2011 IEEE Workshops of International Conference on Advanced Information Networking and Applications (WAINA), March 22-25, pp. 837 $842(2011)$

19. Zheng, H., Boyce, J.: An improved UDP protocol for video transmission over Internet-towireless networks. IEEE Transactions on Multimedia 3(3), 356-365 (2001)

20. Zilly, F., Müller, M., Eisert, P., Kauff, P.: The Stereoscopic Analyzer - An Image-Based Assistance Tool for Stereo Shooting and 3D Production. In: ICIP 2010 - IEEE International Conference, Hong Kong (September 2010) 